\title{
Structural Analysis of the Catalytic Core of Human Telomerase RNA by FRET and Molecular Modelingt,,‡
}

\author{
Gérald Gavory $\nabla, \|$, Martyn F. Symmons $§, \|$, Yamuna Krishnan Ghosh ${ }^{\nabla}$, David Klenerman ${ }^{\nabla}$, \\ and Shankar Balasubramanian ${ }^{*}, \nabla$ \\ $\nabla$ University Chemical Laboratories, Lensfield Road, Cambridge CB2 1EW, U.K. \\ $\S$ Department of Biochemistry, University of Cambridge, Tennis Court Road, Cambridge CB2 \\ 1GA, U.K.
}

\section{Abstract}

Telomerase is the ribonucleoprotein reverse transcriptase involved in the maintenance of the telomeres, the termini of eukaryotic chromosomes. The RNA component of human telomerase (hTR) consists of 451 nucleotides with the $5^{\prime}$ half folding into a highly conserved catalytic core comprising the template region and an adjacent pseudoknot domain (nucleotides 1-208). While the secondary structure of hTR is established, there is little understanding of its three-dimensional (3D) architecture. Here, we have used fluorescence resonance energy transfer (FRET) between fluorescently labelled peptide nucleic acids, hybridized to defined single stranded regions of full length hTR, to evaluate long-range distances. Using molecular modeling, the distance constraints derived by FRET were subsequently used, together with the known secondary structure, to generate a 3D model of the catalytic core of hTR. An overlay of a large set of models generated has provided a low-resolution structure $(6.5-8.0 \AA$ ) that can readily be refined as new structural information becomes available. A notable feature of the modeled structure is the positioning of the template adjacent to the pseudoknot, which brings a number of conserved nucleotides close in space.

Telomerase is the DNA polymerase responsible for the maintenance of the telomeres, the nucleoprotein structures that cap the ends of eukaryotic linear chromosomes. The telomerase enzyme complex is a ribonucleoprotein that minimally comprises an RNA component and a reverse transcriptase protein component $(1,2)$.

The secondary structure of the 451 nucleotide human telomerase RNA (hTR) has been established on the basis of phylogenetic and biochemical footprinting analyses (Figure 1) (3, 4). Although there is considerable variability in the sequence and length of telomerase RNAs across species, there are numerous structural features that appear to be conserved (5, 6). Mutagenesis analyses have demonstrated that the domain-spanning nucleotides 1-208 of hTR are critical for catalytic activity (7), whereas the remaining RNA (nt 209-451) is considered to be primarily involved in protein-RNA interactions (CR4-CR5) and biogenesis

\footnotetext{
$\dagger^{\dagger}$ This work was supported by the BBSRC.

†The atomic coordinates of the final model have been deposited in the Nucleic Acid Database (entry id 2INA). (C) 2006 American Chemical Society

`To whom correspondence should be addressed. Tel: +44-1223-336347. Fax: +44-1223-336362. E-mail: sb10031@ @am.ac.uk.. "G.G. and M.F.S. contributed equally to this work.

SUPPORTING INFORMATION AVAILABLE

Biophysical details of PNAs1-3 binding to hTR. The multimerisation state of hTR. CD spectra of hTR in the presence and absence of hybridised PNA. A flow diagram to explain the molecular modelling approach. Details of the cluster II models of hTR and the variation between clusters I and II. This material is available free of charge via the Internet at http://pubs.acs.org.
} 
of hTR in vivo (box H/ACA domain) $(8,9)$. The template region of the RNA comprises a short sequence complementary to the telomeric repeat and serves a catalytic role directing the sequence of newly synthesised telomeric repeats (10). The P1a and P1b helices are formed by long-range base-pairing interactions that enclose the template and pseudoknot. In particular, helix P1b is responsible for defining the $5^{\prime}$ boundary during repeat addition (11). The pseudoknot domain comprises the $\mathrm{P} 3$ helix, the structural helix P2b and the two loops $\mathrm{J} 2 \mathrm{a} / 3$ and $\mathrm{J} 2 \mathrm{~b} / 3$. Two additional structural helices P2a1 and P2a present in hTR appear not to be conserved. Functional analysis of the pseudoknot, and mutated derivatives, has demonstrated that stable base pairing within a static P3 helix is a critical requirement (12). It has been proposed that both the pseudoknot and template elements are recognized by the hTERT protein component (5).

It has been shown in vitro that hTR has the ability to dimerize via RNA interaction sites that include the P3 helix (13), the P1 helix (14), and the J7b/8a loop (15, 16). Complementation studies suggest that an hTR dimer may be functionally relevant in the telomerase complex $(13,14)$. However, there is no direct structural evidence for the hTR dimer in the telomerase complex. It has been observed that Tetrahymena telomerase can function as a monomer (17), suggesting that RNA dimer formation is not a general functional requirement for the telomerase mechanism.

Elucidation of the three-dimensional (3D) structure of hTR is an important step toward improving our understanding of human telomerase. Approaches for the analysis of the global architecture of large RNA molecules are challenging. High-resolution structure determination of large hTR components by either X-ray crystallography or NMR spectroscopy has thus far been elusive. However, detailed structures of small fragments of hTR have been determined by NMR spectroscopy (18-22). In this study, we developed and exploited a methodology based on labeled hybridization probes and fluorescence resonance energy transfer (FRET) to map long-range distances within nucleotides 1-208 of full-length hTR in its monomeric state. These data were used in conjunction with known constraints from the secondary structure to generate a 3D structural model of the catalytic core of hTR. This study reveals insights into the global architecture of hTR and provides a basis for understanding the spatial relationships between the folded elements of the RNA.

\section{MATERIALS AND METHODS}

\section{RNA Preparation}

Full-length telomerase RNA was prepared from BamHI linearized pUC18-hTR+1 plasmid, which carries hTR cDNA immediately downstream from the T7 promoter (23). hTR was denatured at $90{ }^{\circ} \mathrm{C}$ for $5 \mathrm{~min}$ and snap-cooled on ice before use to minimize multimerization, as reported by others for the study of RNA structure in HIV-1 (24) and telomerase $(18,19)$. The integrity and purity of the transcripts was determined by agarose gel electrophoresis and staining with ethidium bromide.

\section{PNA Synthesis and Labeling}

PNA oligomers were prepared by standard Fmoc methods (25) and singly labeled by coupling with $N$-hydroxy succinimidyl esters of fluorescein $(\mathrm{Fl})$ or tetramethyl-rhodamine (TMR) to either the $\mathcal{\varepsilon}$-amino group of a $C$-terminal lysine residue or directly to the $N$ terminus of the PNA. $C$-terminal labeling was achieved on TGA resin preloaded with FmocLys(Mtt)-OH amino acid (Novabiochem) after the removal of the Mtt protecting group in DCM/TFA/TIS (97:1:2). $N$-terminal labeling was performed on TGA resin preloaded with Fmoc-Lys(Boc)-OH following PNA synthesis and removal of the terminal Fmoc group. HPLC purified PNAs were characterized by electrospray mass spectrometry, and the 
labeling extent (>95\%) was verified by absorption measurements at 260, 498, and $560 \mathrm{~nm}$. Sequences of the PNA hybridization probes were $\mathrm{NH}_{2}$-CCCTCCGCAACCC-

Lys(PNA1), $\mathrm{NH}_{2}$-CAGTTAGGGTTAG-Lys (PNA2), and $\mathrm{NH}_{2}$-AATGAACGGTGGA-Lys (PNA3), each of which was unique for its respective target site with no potential secondary binding site with either full complementarity or a single mismatch.

\section{Fluorescence Measurements}

Steady-state fluorescence emission spectra were collected at $4{ }^{\circ} \mathrm{C}$ on an Amico Bowman Series 2 spectrofluorometer (Amico) using a $10 \mathrm{~mm}$ optical path quartz fluorescence cell (Hellma). Emission spectra were collected between 510 and $700 \mathrm{~nm}$ unless otherwise stated with a $5 \mathrm{~nm}$ monochromator slit width for both the excitation and emission. Excitation wavelengths were 498 and $560 \mathrm{~nm}$ for fluorescein and TMR, respectively. The absorbance of all solutions was below 0.015 at the excitation wavelengths.

\section{Determination of Dissociation Constants}

Dissociation constants $\left(K_{\mathrm{d}}\right)$ were determined by incubating a fixed concentration of hTR (10 $\mathrm{nM}$ ) with increasing concentrations of labeled PNA from 0.05 to $6 \mu \mathrm{M}$ in phosphate buffer (10 mM sodium phosphate ( $\mathrm{pH} 7.0$ ), $100 \mathrm{mM} \mathrm{NaCl}$, and $1 \mathrm{mM} \mathrm{MgCl}_{2}$ ). Following incubation at $20^{\circ} \mathrm{C}$ for $12 \mathrm{~h}$, excess PNA was removed by gel filtration using MicroSpin S-400 HR columns (Amersham Biosciences). Emission spectra were immediately recorded and the emission maxima measured at $525 \mathrm{~nm}$ or $585 \mathrm{~nm}$ for the fluorescein- and TMRlabeled hTR complexes, respectively. Binding curves were obtained and the data fitted to eq 1

$$
\theta=[\mathrm{PNA}] /\left(K_{\mathrm{d}}+[\mathrm{PNA}]\right)
$$

where $\theta$ represents the fractional saturation of hTR (i.e., fraction of hTR bound to PNA), and [PNA] denotes the PNA concentration. $\theta$ and [PNA] were inputs to the nonlinear regression, whereas $K_{\mathrm{d}}$ was an unconstrained output.

\section{FRET Experiments}

hTR $(0.5 \mu \mathrm{M})$ was mixed with a 10-fold molar excess of fluorescein-labeled PNA in phosphate buffer (10 $\mathrm{mM}$ sodium phosphate ( $\mathrm{pH} 7.0), 100 \mathrm{mM} \mathrm{NaCl}$, and $1 \mathrm{mM} \mathrm{MgCl} 2$ ) followed by the addition of the second unlabeled or TMR-labeled PNA (10-fold molar excess). Following incubation at $20^{\circ} \mathrm{C}$ for $12 \mathrm{~h}$, the labeled hTR complex was separated from excess PNA using the MicroSpin S-400 HR columns and the fluorescence emission spectrum immediately recorded as described above. The efficiency of energy transfer $(E)$ was determined by the quenching of the donor fluorescence emission using the following equation $(26,27)$ :

$$
E=1-\left(I_{\mathrm{DA}} / I_{\mathrm{D}}\right)
$$

where $I_{\mathrm{DA}}$ and $I_{\mathrm{D}}$ are the integrated fluorescence intensities of the donor in the presence and absence of the acceptor, respectively (integration of $\pm 10 \mathrm{~nm}$ around the maxima was applied). The interfluorophore distance $(R)$ was then calculated from

$$
R=R_{0}(1 / E-1)^{1 / 6}
$$

where $R_{0}$ is the Förster critical distance at which the energy transfer $E$ is $50 \%(26) . R_{0}$ was determined for each FRET pair using 


$$
R_{0}=9.7910^{3}\left(\eta^{-4} \kappa^{2} \varphi_{\mathrm{D}} J_{\mathrm{DA}}\right)^{1 / 6}(\text { in } \AA)
$$

where $\eta$ is the refractive index of the medium (1.33 for aqueous solutions), $\kappa^{2}$ is the dipolar orientation factor assumed to be $2 / 3$ on the basis of the random tumbling of the fluorophores, $\varphi_{\mathrm{D}}$ is the quantum yield of the donor in the absence of acceptor, and $J_{\mathrm{DA}}$ is the spectral overlap integral between the donor emission and acceptor absorption and was calculated as described previously $(28,29)$.

\section{Computer Modeling}

Modeling was performed using the molecular dynamics program CNS v1.1 (30). The initial tertiary model was interactively built from elementary constituents, including helices, bulges, and loops, based on the secondary structure previously published (3, 4). Canonical A-form RNA helices were used for the duplex regions, whereas the remaining elements were derived from similar RNA motifs present in the Structural Classification of RNA (SCOR) database (31). On the basis of the structural similarities between PNA/RNA and RNA/RNA duplexes, PNA probes were modeled as RNA (32). All elements were manipulated and subsequently assembled into a 3D structure with QUANTA (Accelrys) and XFit (33). Simulated annealing and energy minimization were performed with restraints of secondary structure, including hydrogen-bonding, base pair and nucleobase planarity and dihedral angle restraints $(a, \beta, \gamma, \delta, \varepsilon, \xi$, and $\chi$ ) derived from the Nucleic Acid Database (34).

In addition, the models were required to satisfy the constraints of the experimentally determined FRET distances. Additional end-to-end restraints were also used to enforce Aform conformation of the helices during simulated annealing. The structures were calculated starting from randomized conformations of the secondary structures, and following standard CNS protocols for simulated annealing. The structures were ranked by lowest energy and the top 5\% (i.e., 45 models), lacking major distance ( $>0.5 \AA$ ) and dihedral violations $\left(>5^{\circ}\right)$, were selected as members of the final ensemble. These structures were then refined further using the CHARMm forcefield, superimposed, and classified to indicate the average position of helix axes in the global structure. The resulting models were subjected to a final refinement using the energey minimization with CNS to ensure correct geometry and stereochemistry. Idealized geometric cylinders were fitted to the hTR secondary structures, and the RMSD of cluster midpoints were calculated from the coordinates with Mathematica routines. These cylinders were displayed directly using PyMOL (http://

pymol.sourceforge.net), whereas representations of the model backbone and basepairs were produced by the nuccyl script (http://www.biosci.ki.se/groups/ljo/software/nuccyl.html) before display with PyMOL. The coordinate and restraints files used to generate the final model is available from the Nucleic Acid Database (34) (entry id 2INA).

\section{RESULTS AND DISCUSSION}

\section{Experimental Design}

FRET has been used in many fields of structural biophysics as a molecular ruler. The technique is well suited to determine distances in the 10-100 $\AA$ range and has been used extensively to investigate the folding of various complex RNA structures (35-38). In particular, FRET measurements have been combined with molecular modeling to generate global structures of biological macromolecules (39). In this study, we used FRET to determine long-range distance information between distal parts within the core structure (nt 1 to 208) of full-length hTR (451 nt). The application of FRET requires the presence of a donor and an acceptor fluorophore in defined locations within the structure. Although there 
are established strategies for directly incorporating labels into small nucleic acid fragments, dual-labeling of the RNA component of human telomerase represented a considerable challenge. DNA-hybridization probes have been previously employed in cross-linking studies to investigate the structure of large RNAs such as the ribosome $(40,41)$. We have adapted this approach and used fluorescently labelled 13 mer peptide nucleic acids (PNA) as site-specific probes of hTR. More precisely, we utilized pairs of singly labelled PNA probes comprising a donor (fluorescein) and acceptor (TMR). Each pair of probes was complementary to specific single stranded regions of hTR, enabling the FRET efficiency to be measured and the associated distances to be determined.

\section{Design and Binding Characteristics of PNA Probes}

The PNAs were designed to target regions of hTR known to be single stranded from phylogenetic and biochemical mapping analyses $(3,4)$ and also shown to be accessible for hybridization (42). The target regions were the extreme $5^{\prime}$ end of hTR (nt 1-13; targeted by PNA1), the template region, adjacent nucleotides (nt 44-56; targeted by PNA2), and the pseudoknot loop J2a/3 (nt 146-158; targeted by PNA3) (Figure 1). The PNAs were each synthesized and singly labeled at the $C$ - or $N$-terminus with either fluorescein (donor) or TMR (acceptor) fluorophores (Materials and Methods). We used PNA oligomers in this study owing to their superior hybridization affinity and sequence specificity relative to DNA $(43,44)$. The convention adopted here to designate a particular PNA probe is based on the region targeted plus the identity and point of attachment ( $N$ - or $C$-terminus) of the fluorophore. For example, PNA2nTMR refers to the PNA complementary to the template domain and labeled at the $N$-terminus with TMR.

The thermal stability of the PNA hybridization probes were first investigated by UV-melting experiments with complementary DNA, which indicated high thermal stability $\left(T_{\mathrm{m}}>65^{\circ} \mathrm{C}\right)$ for all PNA/DNA heteroduplexes with little stabilization/destabilization effect from the fluorophores (Supporting Information). The affinity of the PNA probes for native hTR was subsequently determined by fluorescence titration of labeled PNA with hTR, whereby the hTR-PNA complex was separated and quantitated for fluorescence (Materials and Methods). Our results indicated dissociation constants $\left(K_{\mathrm{d}}\right)$ no higher than $300 \mathrm{nM}$ (Table 1 ), suggesting that the PNAs have high binding affinities for their respective targets.

It has been reported that hTR has a capacity to form dimers (13-16). This was an important issue for consideration in FRET studies because it would complicate the interpretation of data resulting from two pairs of FRET probes in a dimeric RNA-RNA system. Our preparation of dual-labeled hTR was predominantly monomeric, with very low levels $(<10 \%)$ of dimeric species, as judged by nondenaturing gel electrophoresis (Supporting Information). We have also considered the possibility of perturbation in the RNA structure induced by the PNA probes. However, previous studies have indicated that hybridization of PNA1 or PNA3 to hTR (pre and postassembly with hTERT) does not significantly alter the catalytic activity of human telomerase (42), suggesting that RNA retains its native conformation and can associate with hTERT to produce a functional complex. Furthermore, the target site for PNA2 is the natural DNA substrate hybridization site, which must accommodate hybridization within a functional, folded structure. To provide further support, we employed circular dichroism spectroscopy, which has been used extensively to characterize the tertiary folding of complex RNA molecules $(45,46)$. The circular dichroism spectra we recorded for hTR and hTR-PNA complexes, respectively, were identical within experimental uncertainty limits (Supporting Information), which also supports a near native global structure for the preparation of monomeric hTR. Although we cannot rule out minor disturbances in the structure, our data, combined with the studies of others (42), suggests 
that there are no significant changes in the global structure of hTR upon hybridization with the PNAs.

\section{FRET Analysis}

FRET is the process by which excited-state energy is transferred nonradiatively from the donor (fluorescein) to the acceptor (TMR) chromophore. This process produces a decrease in the donor fluorescence emission that can be readily measured (47). hTR $(0.5 \mu \mathrm{M})$ was incubated with 10-fold molar excess of both the fluorescein and TMR-labeled PNAs. Following incubation, the dual-labeled hTR complex was purified by size-exclusion chromatography, and the fluorescence spectra were immediately recorded (details in Materials and Methods). Figure 2 shows typical data from a FRET experiment, using the PNA2nTMR/PNA3cFI pair, revealing the associated changes in fluorescein emission observed in the presence or absence of the acceptor probe. In this case, the addition of PNA2nTMR (acceptor probe) caused a 65\% reduction of fluorescein fluorescence, with the concomitant increase in TMR acceptor fluorescence, confirming the occurrence of FRET. Similar experiments were performed for a series of FRET pairs and the energy transfer efficiency $(E)$ was determined using eq 2 (Table 2). The interfluorophore distances $(R)$ were subsequently calculated for each FRET pair using eq 3 (Table 2 ). $R_{0}$ values were determined for each donor/acceptor pair from eq 4 (Table 2), assuming for all cases, a value of $\kappa^{2}=2 / 3$ based on the low fluorescence anisotropy $(r)(r<0.15$; data not shown), indicating free rotational mobility of all of the fluorophores during the lifetime of the excited state (47). Such an assumption has been previously validated in a variety of experimental systems (28). The calculated values of $R_{0}$ are also consistent with reported values for the Fl/TMR molecular pair $(48,49)$.

\section{hTR Modeling and Energy Minimization}

We were interested to know whether a molecular model could be constructed that would satisfy the FRET measurements along with the known secondary structure of hTR, without geometrical violations or steric clashes. The overall strategy for the modeling of hTR was as follows (see flow diagram in Figure 5, Supporting Information). The core structure of hTR was first divided into elementary motifs, helices, internal loops, and bulges. These elements were subsequently assembled, where appropriate, using similar examples from the SCOR database (31) to generate an initial tertiary model of the RNA. Structural constraints derived from both the FRET analysis and the secondary structure were imposed by applying a series of distances, bonds, angles, and torsional restraints. These were applied to a series of randomly generated starting conformations of secondary structure elements by simulated annealing using CNS. If no structural information was available about a nucleotide, it was defined as being in a free conformation, constrained only by its primary structure. The same strategy was used for internal loops and bulges. Following energy minimization, the resulting structures were ranked by lowest energy and the top 5\% (45 structures), which satisfied all of the input restraints including the FRET-derived distance constraints, were selected for the structural analysis.

At the current level of resolution, the molecular modeling results are most usefully compared in the context of helix spatial arrangement and distribution. In order to assess the degree of structural convergence of the models generated, they were superimposed, maximizing overlap of the helices (P1a/b, P2a1, P2a/b, and P3). Sets of structures were then defined on the basis of both the positions and orientations of these helices and the PNA/ RNA heteroduplexes. Two sets of structures (clusters I and II), encompassing $75 \%$ of the ensemble emerged from this classification. The resulting superimposition for structures in cluster I (the most populated with 21 coordinate sets) is shown in Figure 3a. For clarity, the models are depicted as a collection of geometric cylinders, each representing helices P1a/b, 
$\mathrm{P} 2 \mathrm{a} 1, \mathrm{P} 2 \mathrm{a} / \mathrm{b}$, or $\mathrm{P} 3$ as indicated. The overlay revealed a consistent spatial arrangement of the structural elements with a mean root-mean-square deviation (RMSD) value for the positions of helix midpoints of $6.5 \AA$ (Figure $3 \mathrm{~b}$ ). Comparison of all structures, from clusters I and II, revealed a slightly broader distribution and angular displacement of the core helices (mean RMSD of $8.0 \AA$ ). The global architecture and spatial arrangement of core helices for both clusters was, however, similar (Supporting Information, Figures 6 and 7). Positions of the helices formed by PNA1-3 and complementary sequences exhibited higher RMSD values (13 $\AA$ ) compared with those of the core elements. This variation in the helices modeling the PNA/RNA region are likely to have resulted from the inherent flexibility and higher degree of freedom of adjacent single-stranded linkers during the refinement protocol.

Although the structural core of the models is conserved from cluster I to cluster II, there is a difference in the position of the template/PNA2 element relative to the pseudoknot (Figure 3 a vs Supporting Information, Figure 6). In cluster II, the template is more buried in the structure, close to the P1b P3 interface. Steric hindrance in this region appeared to be the cause of a generally higher energy of cluster II models. On the basis of the significant degree of similarity in the positioning of helices within the clusters, we then selected the lowest energy model of cluster I for further refinement. The structure was subjected to two separate rounds of energy minimization (each with 200 iterations), which included electrostatic terms using the CHARMm energy fields. This produced a final structure (Figure 4), which had no experimental restraints violation or van der Waals clashes, and exhibited an average RMSD from ideal geometries for the bonds and angles no greater than $0.003 \AA$ and $0.83^{\circ}$, respectively. The final structure had an RMSD from the original annealed model of $0.51 \AA$ and a calculated free energy value of $-2059 \mathrm{kcal} / \mathrm{mol}$. Although this favorable energy calculation does not in itself validate the model, it strongly precludes the possibility of a structure that could never form under normal conditions.

\section{Comparison with the Pseudoknot NMR Structure}

During the preparation of this manuscript, the solution structure of a $47 \mathrm{nt}$ pseudoknot model derived from hTR was solved by NMR (22). The construct used for that study suggested functionally important tertiary interactions between loop $\mathrm{J} 2 \mathrm{~b} / 3$ and the major groove of helix $\mathrm{P} 3$, and between loop $\mathrm{J} 2 \mathrm{a} / 3$ and the minor groove of helix P2b (22). We found that the pseudoknot structure predicted by our modeling compared favorably to that derived by NMR. The backbone RMSD between the two structures was calculated to be $5.8 \AA$, 11 which is within the resolution of our model (6.5-8.0 $)$ and provides some independent validation for our study. Subsequently, we have further refined our working model to take account of the additional interactions described in the NMR structure. The refinement involved the single-stranded poly $\mathrm{U}$ sequence in loop J2b/3 (nt 99-102) and poly A in loop $\mathrm{J} 2 \mathrm{a} / 3$ (nt 171-173), being repositioned into the groove of the structure to form the base triplex interactions described by Feigon and co-workers (Figure 5; 22). The backbone RMSD between the refined model of the pseudoknot and the NMR structure was calculated to be $5.5 \AA .{ }^{1}$ This refinement resulted in the same overall topology of the catalytic core of hTR and suggests that our model can accommodate further refinement as new structural information becomes available.

\section{Features of hTR Core Architecture}

The final model derived from the combination of experimental FRET distance constraints and modeling predicts several noteworthy features. The FRET restraints produce hTR models with regions of open structure. This is in part due to helices P1 and P2a1 being

\footnotetext{
${ }^{1}$ The RMSD calculation was performed on nucleotides 93-121 and 171-183 of hTR and excluded the loop containing nucleotides 122-170, which are not native to hTR, and was a particular feature of the RNA used in the NMR studies.
} 
connected by relatively long and malleable single-stranded regions ( $\mathrm{J} 2 \mathrm{a} / 3$ and the template region), which confer an inherent breathability to the core domain of hTR. This feature is unusual compared with other large RNA components found in RNPs such as ribonucleases (50), where the RNA structure is typically more compact. A potential explanation is the requirement for telomerase RNA to accommodate the large catalytic subunit protein TERT and also serve as a scaffold for numerous other associated proteins (51). In this respect, the openness of the structure may enhance RNA accessibility and facilitate assembly and maturation of the telomerase holoenzyme complex. Another outcome of the FRET measurements is the requirement for the $5^{\prime}$ end of the $\mathrm{J} 2 \mathrm{a} / 3$ loop (and stem of helix P2a1) to be near to the P1 helix, contrary to the perspective given by the conventional extended 2D map of hTR. This appears to be accommodated by the acute bend (approximately $70^{\circ}$ ) between helices P2a and P2b induced by the asymmetry in the intervening bulge, which comprises $2 \mathrm{nt}$ on one side and $7 \mathrm{nt}$ on the other. The structure also reveals that bending angles between other pairs of helices help satisfy the FRET distance constraints. The bulge connecting helices P2a and P2a1 accommodates a bend of $\sim 45^{\circ}$, and the bulge connecting $\mathrm{P} 1 \mathrm{a}$ and P1b produces a bend of $\sim 70^{\circ}$.

The template/PNA2 helix extends between helices P1b and P2a1 and lies at an angle of approximately $45^{\circ}$ to the adjacent $\mathrm{P} 3$ helix of the pseudoknot in the lowest energy example (Figure 4a). Although there is some variation in the angle of the template/PNA2 helix within the cluster I models (Figure 3a), it generally lies at an approximately $90^{\circ}$ angle relative to helix P2a1, as reflected in the refined structure (Figure 4). With the template/PNA2 in this position, FRET restraints between PNA2 and PNA3 terminal groups constrain the J2a/3 linker to depart on the opposite side of helix 2a1 template/PNA2. It is also noticeable that the PNA1 helical segment is constrained by the FRET measurements to sit at right angles to the adjacent helix P1a. Although this appears unsupported by molecular contacts in the 1208 nucleotide domain of hTR, it may pack adjacent to other structural elements present in the experiments (i.e., in nucleotides 209-451), which were not subject to the current FRET measurements because of the lack of appropriate hybridization sites for the PNA probes.

In considering the molecular mechanism of human telomerase, we believe the openness and the apparent structural flexibility of the RNA in the template region is significant. The structure in Figure 1 shows a short RNA double helix (labeled PNA 2) in the template region, where one strand (in this case RNA) occupies the space that would normally be taken by the telomeric DNA substrate. The $3^{\prime}$ end of the substrate (shown by the green sphere in Figure 4) is base-paired with the $5^{\prime}$ end of the catalytic template; thus, the complex is representative of a telomeric substrate that has been extended by one hexanucleotide repeat immediately prior to either translocation or dissociation. This $3^{\prime}$ end is positioned close to the helix P1b but the connecting 8nt template linker is extended to allow the $3^{\prime}$ end to be presented to the periphery of the RNA core (Figure $4 \mathrm{~b}$ ). The extension of this linker may help define the template boundary.

A consequence of the bending induced by bulges, as described earlier, is the positioning of the ends of helices P2a1 and P1b, which causes the single-stranded template to have more freedom than would be the case if it were more extended. This is consistent with the requirement for the catalytically functional RNA template to undergo an overall translational motion of $\sim 20 \AA$ relative to the protein active site, and also most likely the anchor points on the RNA where RNA-protein contacts are maintained during the process.

Another notable feature of the structure is the convergence of the conserved nucleotides (labeled as red spheres in Figure 4) toward the active site of the model. Phylogenetic comparisons reveal that, although most structural elements are highly conserved in the RNA component of vertebrate telomerases characterized, only a small fraction of nucleotides are 
universally conserved among vertebrates (15\% for the core structure of hTR) (3). The conservation of the pseudoknot in vertebrates and lower eukaryotes is also striking (6). It is reasonable to anticipate that the active site of telomerase comprises conserved nucleotides located in various regions of the RNA that may be expected to be close in the tertiary structure. The position of the pseudoknot adjacent to the template sequence and resulting spatial convergence of the most evolutionarily conserved residues raises the possibility that the pseudoknot may form part of the enzyme active site.

Our model of hTR provides a first approximation of the global catalytic core structure of hTR. Although we cannot totally rule out that the structure of hTR is altered upon complexation with hTERT, we predict that many structural features programmed into the sequence of hTR are likely to exist in the functional complex. Owing to the practical difficulties associated with the efficiency of hTERT expression and the assembly of human telomerase complex, it is not yet possible to carry out ensemble FRET studies on the human telomerase complex. We intend to address this limitation by employing single molecule fluorescence methods.

\section{Acknowledgments}

We thank Drs. Katherine Stott and Bill Broadhurst for helpful discussions on modeling, Dr. Sven Sewitz for proofreading the manuscript, and Graham Eliff for computer support. S.B. is a BBSRC Career Development Research Fellow.

\section{REFERENCES}

1. Weinrich SL, Pruzan R, Ma LB, Ouellette M, Tesmer VM, Holt SE, Bodnar AG, Lichtsteiner S, Kim NW, Trager JB, Taylor RD, Carlos R, Andrews WH, Wright WE, Shay JW, Harley CB, Morin GB. Reconstitution of human telomerase with the template RNA component hTR and the catalytic protein subunit hTRT. Nat. Genet. 1997; 17:498-502. [PubMed: 9398860]

2. Beattie TL, Zhou W, Robinson MO, Harrington L. Reconstitution of human telomerase activity in vitro. Curr. Biol. 1998; 8:177-180. [PubMed: 9443919]

3. Chen JL, Blasco MA, Greider CW. Secondary structure of vertebrate telomerase RNA. Cell. 2000; 100:503-514. [PubMed: 10721988]

4. Antal M, Boros E, Solymosy F, Kiss T. Analysis of the structure of human telomerase RNA in vivo. Nucleic Acids Res. 2002; 30:912-920. [PubMed: 11842102]

5. Chen JL, Greider CW. An emerging consensus for telomerase RNA structure. Proc. Natl. Acad. Sci. U.S.A. 2004; 101:14683-14684. [PubMed: 15466703]

6. Lin J, Ly H, Hussain A, Abraham M, Pearl S, Tzfati Y, Parslow TG, Blackburn EH. A universal telomerase RNA core structure includes structured motifs required for binding the telomerase reverse transcriptase protein. Proc. Natl. Acad. Sci. U.S.A. 2004; 101:14713-14718. [PubMed: 15371596]

7. Autexier C, Pruzan R, Funk WD, Greider CW. Reconstitution of human telomerase activity and identification of a minimal functional region of the human telomerase RNA. EMBO J. 1996; 15:5928-5935. [PubMed: 8918470]

8. Bachand F, Triki F, Autexier C. Human telomerase RNA-protein interactions. Nucleic Acids Res. 2001; 29:3385-3393. [PubMed: 11504876]

9. Mitchell JR, Cheng J, Collins K. A box H/ACA small nucleolar RNA-like domain at the human telomerase RNA 3' end. Mol. Cell. Biol. 1999; 19:567-576. [PubMed: 9858580]

10. Morin GB. The human telomere terminal transferase enzyme is a ribonucleoprotein that synthesizes TTAGGG repeats. Cell. 1989; 59:521-529. [PubMed: 2805070]

11. Chen JL, Greider CW. Template boundary definition in mammalian telomerase. Genes Dev. 2003; 17:2747-2752. [PubMed: 14630939]

12. Chen JL, Greider CW. Functional analysis of the pseudoknot structure in human telomerase RNA. Proc. Natl. Acad. Sci. U.S.A. 2005; 102:8080-8085. [PubMed: 15849264] 
13. Ly H, Xu LF, Rivera MA, Parslow TG, Blackburn EH. A role for a novel 'trans-pseudoknot' RNA-RNA interaction in the functional dimerization of human telomerase. Genes Dev. 2003; 17:1078-1083. [PubMed: 12730131]

14. Moriarty TJ, Marie-Egyptienne DT, Autexier C. Functional organization of repeat addition processivity and DNA synthesis determinants in the human telomerase multimer. Mol. Cell. Biol. 2004; 24:3720-3733. [PubMed: 15082768]

15. Marie-Egyptienne DT, Cerone MA, Londono-Vallejo JA, Autexier C. A human-Tetrahymena pseudoknot chimeric telomerase RNA reconstitutes a nonprocessive enzyme in vitro that is defective in telomere elongation. Nucleic Acids Res. 2005; 33:5446-5457. [PubMed: 16192571]

16. Ren XJ, Gavory G, Li HT, Ying LM, Klenerman D, Balasubramanian S. Identification of a new RNA: RNA interaction site for human telomerase RNA (hTR): structural implications for hTR accumulation and a dyskeratosis congenita point mutation. Nucleic Acids Res. 2003; 31:6509_ 6515. [PubMed: 14602909]

17. Bryan TM, Goodrich KJ, Cech TR. Tetrahymena telomerase is active as a monomer. Mol. Biol. Cell. 2003; 14:4794-4804. [PubMed: 13679509]

18. Leeper T, Leulliot N, Varani G. The solution structure of an essential stem-loop of human telomerase RNA. Nucleic Acids Res. 2003; 31:2614-2621. [PubMed: 12736311]

19. Leeper TC, Varani G. The structure of an enzyme-activating fragment of human telomerase RNA. RNA. 2005; 11:394-403. [PubMed: 15703438]

20. Theimer CA, Finger LD, Trantirek L, Feigon J. Mutations linked to dyskeratosis congenita cause changes in the structural equilibrium in telomerase RNA. Proc. Natl. Acad. Sci. U.S.A. 2003; 100:449-454. [PubMed: 12525685]

21. Comolli LR, Smirnov I, Xu LF, Blackburn EH, James TL. A molecular switch underlies a human telomerase disease. Proc. Natl. Acad. Sci. U.S.A. 2002; 99:16998-17003. [PubMed: 12482936]

22. Theimer CA, Blois CA, Feigon J. Structure of the human telomerase RNA pseudoknot reveals conserved tertiary interactions essential for function. Mol. Cell. 2005; 17:671-682. [PubMed: 15749017]

23. Gavory G, Farrow M, Balasubramanian S. Minimum length requirement of the alignment domain of human telomerase RNA to sustain catalytic activity in vitro. Nucleic Acids Res. 2002; 30:44704480. [PubMed: 12384594]

24. Lodmell JS, Ehresmann C, Ehresmann B, Marquet R. Structure and dimerization of HIV-1 kissing loop aptamers. J. Mol. Biol. 2001; 311:475-490. [PubMed: 11493002]

25. Simmons CG, Pitts AE, Mayfield LD, Shay JW, Corey DR. Synthesis and membrane permeability of PNA-peptide conjugates. Bioorg. Med. Chem. Lett. 1997; 7:3001-3006.

26. Lakowicz, JR. Principles of Fluorescence Spectroscopy. 2nd ed.. New York: Plenum Publishers; 1999. Energy Transfer; p. 367-394.

27. Walter NG, Burke JM. Fluorescence assays to study structure, dynamics, and function of RNA and RNA-ligand complexes. RNA-Ligand Interact. Part A. 2000; 317:409-440.

28. Xiao J, Singleton SF. Elucidating a key intermediate in homologous DNA strand exchange: Structural characterization of the RecA-triple-stranded DNA complex using fluorescence resonance energy transfer. J. Mol. Biol. 2002; 320:529-558. [PubMed: 12096908]

29. Furey WS, Joyce CM, Osborne MA, Klenerman D, Peliska JA, Balasubramanian S. Use of fluorescence resonance energy transfer to investigate the conformation of DNA substrates bound to the Klenow fragment. Biochemistry. 1998; 37:2979-2990. [PubMed: 9485450]

30. Brünger AT, Adams PD, Clore GM, Delano WL, Gros P, Grosse-Kunstleve RW, Jiang JS, Kuszewski J, Nilges M, Pannu NS, Read RJ, Rice LM, Simonson T, Warren GL. Crystallography \& NMR System: a new software suite for macromolecular structure determination. Acta Crystallogr., Sect. D. 1998; 54:905-921. [PubMed: 9757107]

31. Klosterman PS, Tamura M, Holbrook SR, Brenner SE. SCOR: A structural classification of RNA database. Nucleic Acids Res. 2002; 30:392-394. [PubMed: 11752346]

32. Brown SC, Thomson SA, Veal JM, Davis DG. NMR solution structure of a peptide nucleic-acid complexed with RNA. Science. 1994; 265:777-780. [PubMed: 7519361]

33. McRee DE. XtalView Xfit - A versatile program for manipulating atomic coordinates and electron density. J. Struct. Biol. 1999; 125:156-165. [PubMed: 10222271] 
34. Berman HM, Olson WK, Beveridge DL, Westbrook J, Gelbin A, Demeny T, Hsieh SH, Srinivasan AR, Schneider B. The Nucleic-Acid Database - a comprehensive relational database of 3dimensional structures of nucleic-acids. Biophys. J. 1992; 63:751-759. [PubMed: 1384741]

35. Clegg RM, Murchie AIH, Lilley DMJ. The solution structure of the 4-way DNA junction at lowsalt conditions - a fluorescence resonance energy-transfer analysis. Biophys. J. 1994; 66:99-109. [PubMed: 8130350]

36. Bassi GS, Murchie AIH, Walter F, Clegg RM, Lilley DMJ. Ion-induced folding of the hammerhead ribozyme: a fluorescence resonance energy transfer study. EMBO J. 1997; 16:74817489. [PubMed: 9405376]

37. Walter F, Murchie AIH, Duckett DR, Lilley DMJ. Global structure of four-way RNA junctions studied using fluorescence resonance energy transfer. RNA. 1998; 4:719-728. [PubMed: 9622130]

38. Lafontaine DA, Norman DG, Lilley DMJ. The global structure of the VS ribozyme. EMBO J. 2002; 21:2461-2471. [PubMed: 12006498]

39. van Dijk ADJ, Boelens R, Bonvin AMJJ. Data-driven docking for the study of biomolecular complexes. FEBS J. 2005; 272:293-312. [PubMed: 15654870]

40. Wang R, Alexander RW, VanLoock M, Vladimirov S, Bukhtiyarov Y, Harvey SC, Cooperman BS. Three-dimensional placement of the conserved 530 loop of $16 \mathrm{~S}$ rRNA and of its neighboring components in the 30 S subunit. J. Mol. Biol. 1999; 286:521-540. [PubMed: 9973568]

41. Seo HS, Cooperman BS. Large-scale motions within ribosomal 50S subunits as demonstrated using photolabile oligonucleotides. Bioorg. Chem. 2002; 30:163-187. [PubMed: 12406702]

42. Hamilton SE, Simmons CG, Kathiriya IS, Corey DR. Cellular delivery of peptide nucleic acids and inhibition of human telomerase. Chem. Biol. 1999; 6:343-351. [PubMed: 10375543]

43. Larsen HJ, Bentin T, Nielsen PE. Antisense properties of peptide nucleic acid. Biochim. Biophys. Acta. 1999; 1489:159-166. [PubMed: 10807005]

44. Norton JC, Piatyszek MA, Wright WE, Shay JW, Corey DR. Inhibition of human telomerase activity by peptide nucleic acids. Nat. Biotechnol. 1996; 14:615-61935. [PubMed: 9630953]

45. Pan T, Sosnick TR. Intermediates and kinetic traps in the folding of a large ribozyme revealed by circular dichroism and UV absorbance spectroscopies and catalytic activity. Nat. Struct. Biol. 1997; 4:931-938. [PubMed: 9360610]

46. Vos S, Berrisford DJ, Avis JM. Effect of magnesium ions on the tertiary structure of the hepatitis C virus IRES and its affinity for the cyclic peptide antibiotic viomycin. Biochemistry. 2002; 41:5383-5396. [PubMed: 11969398]

47. Clegg RM. Fluorescence resonance energy-transfer and nucleic-acids. Methods Enzymol. 1992; 211:353-388. [PubMed: 1406315]

48. Toth K, Brun N, Langowski J. Trajectory of nucleosomal linker DNA studied by fluorescence resonance energy transfer. Biochemistry. 2001; 40:6921-6928. [PubMed: 11389607]

49. Hardwidge PR, Wu J, Williams SL, Parkhurst KM, Parkhurst LJ, Maher LJ. DNA bending by bZIP charge variants: A unified study using electrophoretic phasing and fluorescence resonance energy transfer. Biochemistry. 2002; 41:7732-7742. [PubMed: 12056905]

50. Tsai HY, Masquida B, Biswas R, Westhof E, Gopalan V. Molecular modeling of the threedimensional structure of the bacterial RNase pholoenzyme. J. Mol. Biol. 2003; 325:661-675. [PubMed: 12507471]

51. Mergny JL, Riou JF, Mailliet P, Teulade-Fichou MP, Gilson E. Natural and pharmacological regulation of telomerase. Nucleic Acids Res. 2002; 30:839-865. [PubMed: 11842096] 


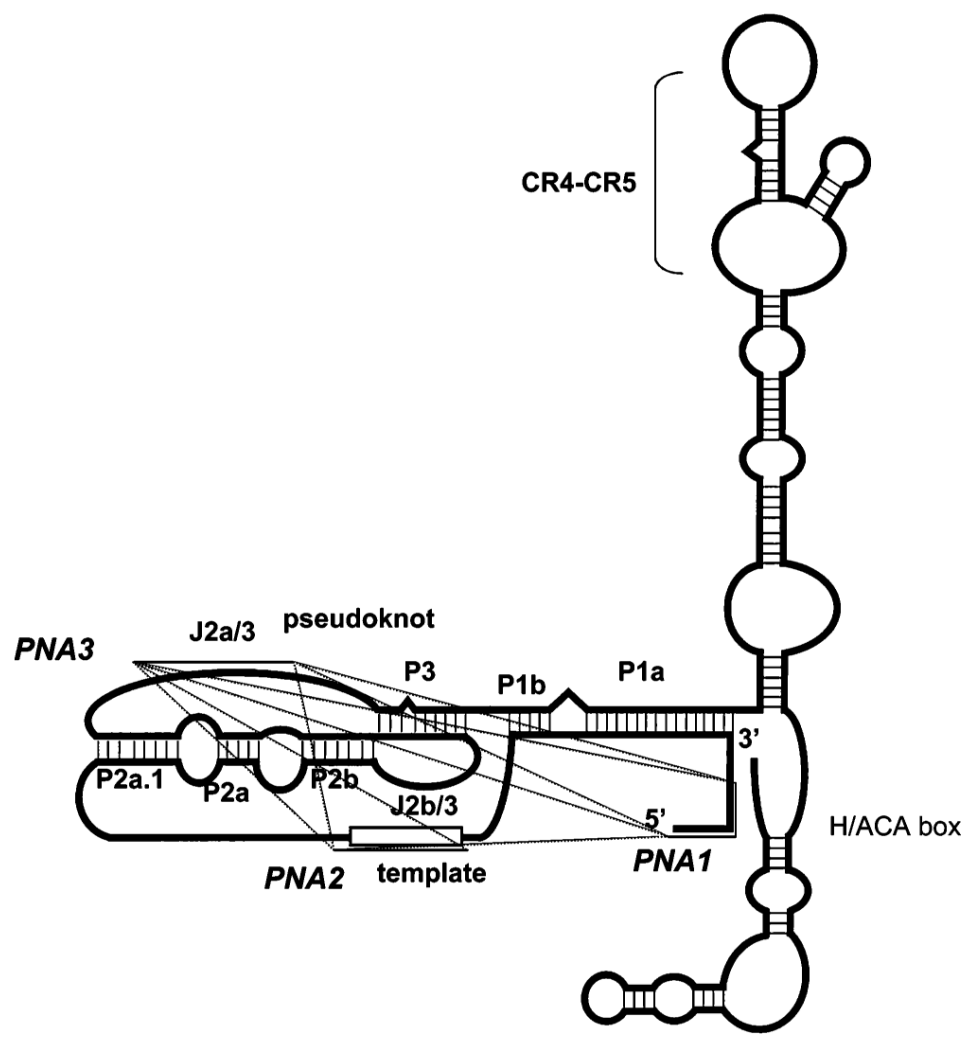

Figure 1.

Secondary structure of human telomerase RNA (hTR) (adapted from refs 3 and 4 (3 and 4)). The underlined sequence around the $5^{\prime}$ end, the template, and in loop J2a/3 of hTR indicate the PNA binding sites (for PNA1, PNA2, and PNA3, respectively). FRET distances determined in this study are indicated by dotted lines. 


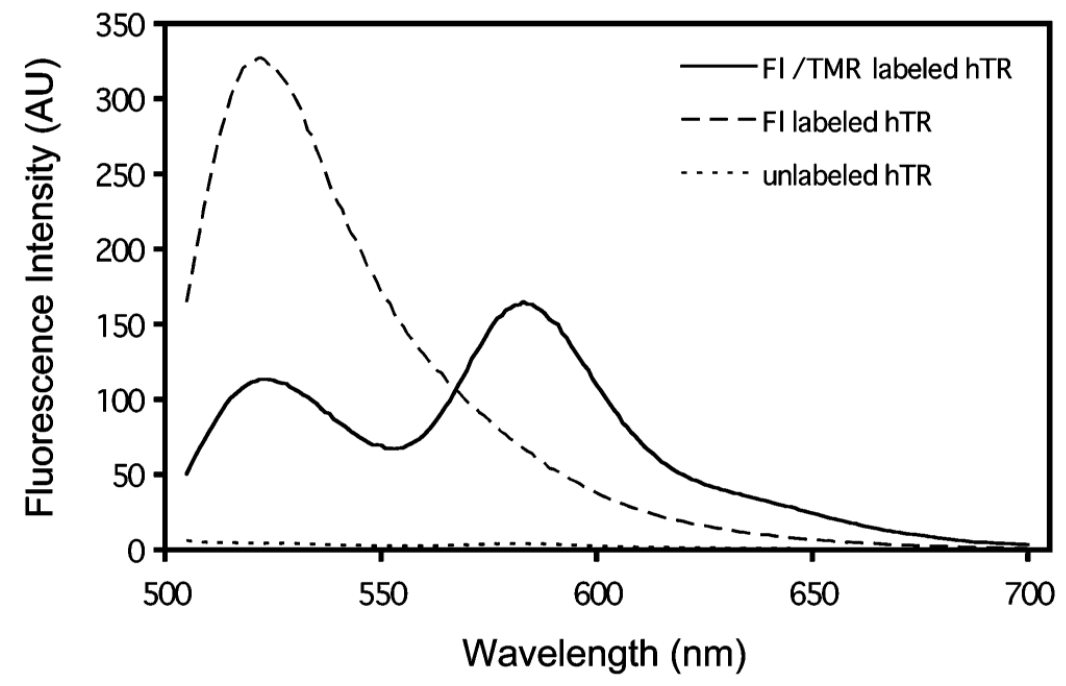

Figure 2.

Fluorescence data for a typical FRET experiment using the PNA2nTMR/PNA3cFl molecular pair. The emission spectra are as follows: fluorescein- and TMR-labeled hTR (donor-acceptor, solid line) and fluorescein-labeled hTR (donor only, broken line). The blank measurement (unlabeled hTR, dashed line) was used for background correction. Transfer efficiency values $(E)$ were calculated using eq 2 . 


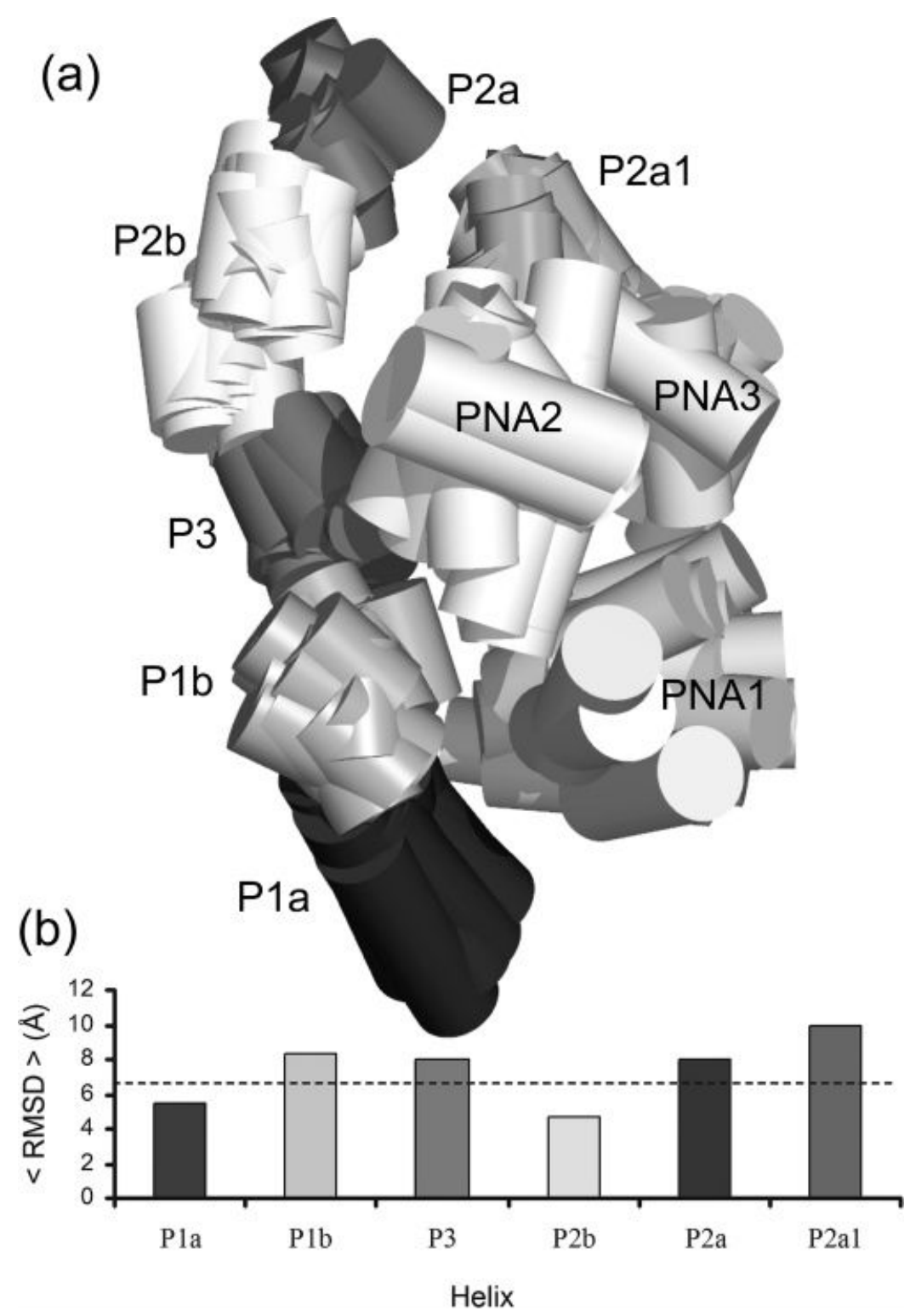

Figure 3.

Superimposition of RNA helices in lowest energy refined models. (a) Superimposition of helices of the catalytic core of human telomerase RNA (cluster I, 21 models). For clarity, the helical elements are displayed as cylinders of appropriate length but with diameter $50 \%$ that of an A-form RNA helix width. (b) Variation in the position of helices between the superimposed structures of cluster I. The variation is expressed as the mean RMSD (< RMSD $>$ ) in the position of a particular helix midpoint relative to the corresponding helix in the lowest energy structure. The average of the RMSD values for all helices $(6.5 \AA)$ is represented by the dotted line. $\mathrm{P} 2 \mathrm{~b}$ was actually fitted to two helical segments whose displacements were averaged for presentation in this graph. 

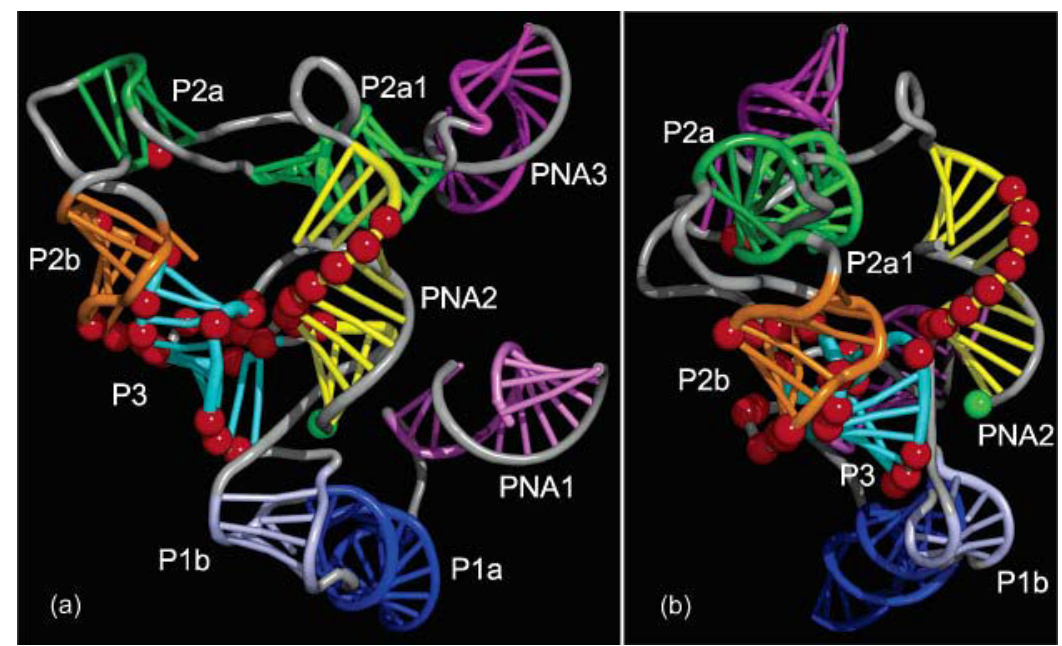

Figure 4.

Schematic representation of lowest energy model. (a) Refined 3D molecular model of nucleotides 1-208 of human telomerase RNA. The RNA backbones are represented as ribbons colored gray for linkers and PNA or distinctive colors for each helix. Base positions in helices are indicated schematically by single colored sticks positioned by the nuccyl program. Nucleotides invariant among all vertebrates are shown as red spheres. The green sphere corresponds to the position of the $3^{\prime}$ end of the RNA representing PNA 2 and, thus, the $3^{\prime}$ end of the DNA primer immediately after a repeat addition but prior to translocation. (b) Alternative view of structure after a $90^{\circ}$ rotation about the vertical axis. 


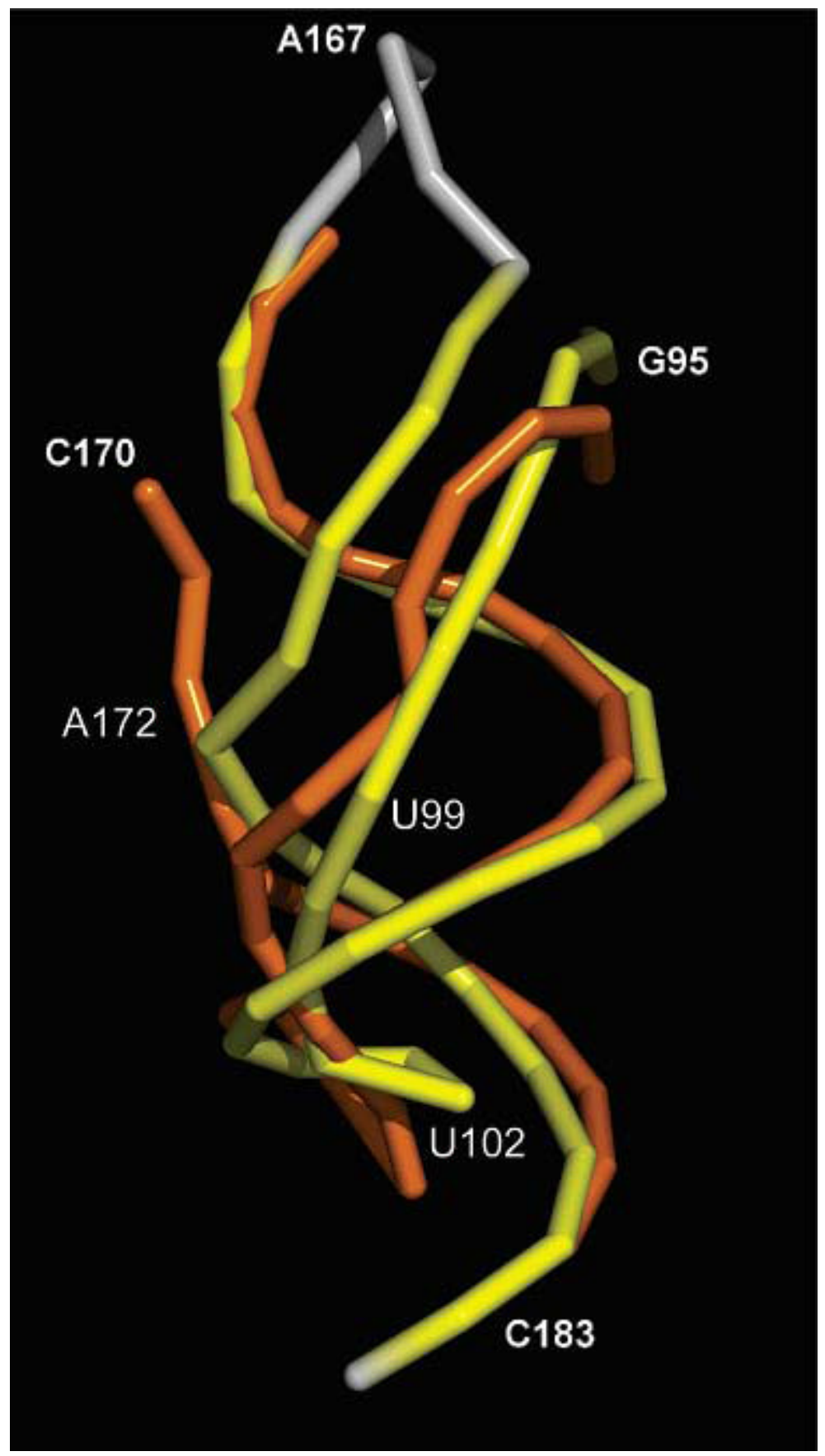

Figure 5.

Comparison of refined pseudoknot with NMR model. Superimposition of the backbone structure of hTR pseudoknot, derived by NMR spectroscopy (yellow), with that of our further refined model (orange). The loop spanning nt 122-170, shown in gray, is a particular feature of the RNA used in the NMR studies (12) and was not included in the RMSD calculation. 


\section{Table 1}

Binding Affinities of PNA Probes for Native hTR

\begin{tabular}{lll}
\hline PNA & target region & $\boldsymbol{K}_{\mathbf{d}}(\mathbf{n M})^{\boldsymbol{a}}$ \\
\hline PNA1 & $5^{\prime}$-end (nt 1-13) & $300 \pm 25$ \\
PNA2 & template (nt 44-56) & $150 \pm 50$ \\
PNA3 & loop J2a/3 (nt 146-158) & $280 \pm 35$ \\
\hline
\end{tabular}

aDissociation constants $\left(K_{\mathrm{d}}\right)$ were determined by fluorimetric titration using PNA1nFl, PNA2nTMR, and PNA3nFl (see Experimental Procedures for details). A typical data set is shown for PNA2nTMR in Supporting Information. $K_{\mathrm{d}}$ values represent the mean of at least two independent experiments. 


\section{Table 2}

Energy Transfer and Distance Measurements

\begin{tabular}{lccc}
\hline FRET pair & $\mathbf{E}^{\boldsymbol{a}}$ & $\boldsymbol{R}_{\mathbf{0}}(\AA)^{\boldsymbol{a}}$ & $\boldsymbol{R}\left(\AA \mathrm{A}^{\boldsymbol{a}}\right.$ \\
\hline PNA2nTMR/PNA3nFl & $0.61 \pm 0.07$ & 61.3 & $56.9 \pm 2.3$ \\
PNA2nTMR/PNA3cFl & $0.73 \pm 0.04$ & 61.4 & $52.0 \pm 1.6$ \\
PNA2nTMR/PNA1cFl & $0.70 \pm 0.03$ & 59.9 & $51.7 \pm 1.2$ \\
PNA2nTMR/PNA1nFl & $0.76 \pm 0.02$ & 60.8 & $50.2 \pm 1.0$ \\
PNA2cTMR/PNA3nFl & $0.49 \pm 0.03$ & 61.3 & $61.7 \pm 1.2$ \\
PNA1nTMR/PNA3nFl & $0.76 \pm 0.01$ & 61.3 & $50.6 \pm 0.6$ \\
PNA1nTMR/PNA3cFl & $0.64 \pm 0.01$ & 61.4 & $55.8 \pm 0.2$ \\
PNA1cTMR/PNA3nFl & $0.50 \pm 0.05$ & 61.3 & $61.3 \pm 1.8$ \\
\hline
\end{tabular}

aEnergy transfer efficiencies $(E)$, Förster distances $\left(R_{0}\right)$, and interfluorophore distances $(R)$ were determined as described in Experimental Procedures. Averages were derived from at least two independent experiments. 